\title{
PROYECTOS FORMATIVOS INTEGRADORES EN LA FORMACIÓN PROFESIONAL
}

\section{Integrating training projects in vocational training}

iD Huerta Rosales Moises

'Universidad Nacional Santiago Antúnez de Mayolo, Perú

Correspondencia:

Dr. Moises Huerta Rosales

mohuereduc@hotmail.com

\section{RESUMEN}

El desarrollo de los proyectos integradores en la formación profesional, tiene como propósito integrar la investigación formativa con las acciones de responsabilidad social, para contribuir a la mejora de la calidad de la formación profesional, en la que la investigación como proceso de exploración y descubrimiento de nuevos conocimientos, contribuye al desarrollo tecnológico que genera tecnologías, procesos o productos, derivando en la innovación tecnología, que permite la transferencia de la tecnología para su usufructo de los mismos en beneficio de la sociedad. Este informe emplea, una metodología de investigación acción orientada a verificar en la práctica la solución de la problemática de aprender a investigar a partir de intervenir en la solución de los problemas del contexto. El resultado refleja que los estudiantes potencian sus capacidades investigativas al mejorar sus capacidades de observación, detección, análisis y uso de la información para traducirla en comunicaciones científicas y mejor aún, si estas responden a buscar la solución de problemas del contexto. En conclusión, la aplicación de los proyectos formativos integradores potencian la capacidad de investigar a partir de la realidad y contribuir a la formación del ser humano investigador con responsabilidad humana, social y ambiental.

Palabras clave: Proyectos formativos integradores, investigación formativa, responsabilidad social

\section{ABSTRACT}

The development of integrative projects in professional training, aims to integrate formative research with social responsibility actions, to contribute to the improvement of the quality of professional training, in which research as a process of exploration and discovery of new knowledge, contributes to the technological development that generates technologies, processes or products, resulting in technological innovation; which allows the transfer of technology for its use for the benefit of society. This report uses an action research methodology aimed at verifying in practice the solution of the problem of learning to investigate from intervening in the solution of the problems of the context. The result is that students enhance their investigative skills by improving their observation, detection, analysis and use of information to translate it into scientific 
communications and better still, if they respond to seek the solution of problems in the context. In conclusion, the application of integrative training projects enhances the ability to investigate from reality and contribute to the training of the human being researcher with human, social and environmental responsibility.

Key words: Integrative training projects, training research, social responsibility.

\section{INTRODUCCIÓN}

\section{La investigación}

La investigación es un proceso formal, sistemático e intensivo del método de análisis, consecuentemente, es la fase más específica del método científico (Best, 1986). Del mismo modo, la investigación es un proceso reflexivo, controlado y crítico que permite descubrir nuevos hechos o datos, relaciones o leyes, en cualquier campo del saber humano (Ander Egg, 2001). Desde otras perspectivas la investigación es un proceso constante de exploración y descubrimientos (Salkind, 2010). Kerlinger (2002), complementa que la investigación científica es sistemática, controlada, empírica y crítico, de proposiciones hipotéticas sobre supuestas relaciones que existen entre los fenómenos naturales.

En síntesis, la investigación es un proceso de exploración y descubrimiento de nuevos conocimientos, construidos en forma sistemática, controlada, empírica y crítica, de proposiciones hipotéticas sobre supuestas relaciones que existen entre los fenómenos naturales, sociales o culturales. La investigación científica es creadora de conocimientos.

\section{Desarrollo tecnológico}

El desarrollo tecnológico es el uso sistemático del conocimiento y la investigación dirigido hacia la producción de materiales, dispositivos, sistemas o métodos incluyendo el diseño, desarrollo, mejora de prototipos, procesos, productos, servicios o modelos organizativos (Concytec, 2017). Es el uso creativo del conocimiento científico en la producción de bienes y servicios. Así, el desarrollo tecnológico es un avance en la eficiencia técnica con la que los recursos son usados en la producción, es decir, permite producir una mayor cantidad de bienes y servicios con los mismos recursos o seguir produciendo lo mismo con menos recursos y costo; generando nuevas tecnologías, procesos o productos. Es un proceso que complementa a la investigación, pues permite convertir los nuevos conocimientos en nuevos procesos o productos; por tanto, el desarrollo tecnológico genera tecnologías, procesos o productos derivados en la obtención de patentes y derechos de propiedad intelectual.

\section{Innovación tecnológica}

Innovación es la generación de un nuevo producto, bien o servicio, un proceso, método de comercialización o método organizativo, en nuevos productos o prácticas internas de la empresa, la organización del lugar de trabajo o las relaciones exteriores. Innovación tecnológica, por su parte, se distingue por una mejora o novedad en las características del desempeño de los productos o servicios, y su aplicabilidad en la práctica dependerá del grado de variabilidad en sus características y su novedad, que constituyan un factor importante en las ventas de una empresa o industria (Concytec, 2017).

La innovación tecnología, implica transferencia de la tecnología para su usufructo en bien de la generación de nuevos procesos o productos que benefician a la sociedad. La innovación tecnológica no es otra cosa que el uso comercial de las patentes y propiedad intelectual para poner al servicio de la comunidad; es decir, transformar las invenciones y descubrimientos en bienes o servicio de uso público.

\section{Investigación formativa}

La investigación formativa, proporciona a los estudiantes un conjunto de estrategias para el desarrollo de sus capacidades investigativas. Son de naturaleza formativa e instrumental y permite al estudiante, prepararse para el desarrollo de la investigación científica de fin de carrera o integrar equipos de investigación. Para Restrepo (2008), 
la investigación formativa no se circunscribe a una metodología científica estandariza, basadas en el rigor metodológico; sino por el contrario, es un proceso didáctico que permite "entrenar" al estudiante en el empleo menos sistemático de búsqueda de información científica, consulta con expertos, hacer construcciones teóricas o modelos conceptuales, el planteamiento de casos, ensayos de prototipos de laboratorio, sondeos de necesidades, análisis de contextos, etc.; con la finalidad de comunicar sus resultados en forma de monografías, ensayos, informes técnicos, tesinas, artículos científicos, etc.

Restrepo (2018); manifiesta que los estudiantes durante la investigación formativa, aplican las actividades básicas que incorporan la lógica de la investigación y aplican el método científico; pero que no implica necesariamente el desarrollo de proyectos de investigación formales ni el hallazgo de conocimientos nuevos ni universales. Es así que la investigación, se lleva a cabo a través del proceso de enseñanza y aprendizaje, el cual puede desarrollarse desde diversas estrategias didácticas, principalmente a través del aprendizaje por descubrimiento, aprendizaje basado en problemas, métodos de proyectos, etc., más proclive a la construcción de conocimientos por parte del estudiante.

Abordar la investigación formativa como una herramienta de formación implica utilizarla como estrategias de enseñanza y aprendizaje; en este contexto, Padilla, Rincón y Buitrago (2015) proponen las siguientes características:

a. Prepara al estudiante en el manejo de herramientas básicas para la investigación: Búsqueda de datos, organización de la información, comunicación de los resultados.

b. Articula el currículo con el desarrollo de procesos de enseñanza y aprendizaje de una o más asignaturas.

c. No se enmarca dentro de una línea de investigación, sino en un programa académico formativo.

d. Los trabajos académicos se desarrollan gradualmente.

e. La complejidad de los trabajos académicos se desarrolla gradualmente: monografías, ensayos, informes técnicos, etc.

f. El objeto de investigación pertenece a un área de saber ya establecido y debe orientarse en forma interdisciplinaria.

g. Las técnicas e instrumentos de investigación se subordinan a las estrategias didácticas.

h. Es una investigación dirigida y orientada por un profesor, como parte de su función docente.

Restrepo (2008), por su parte incorpora las siguientes estrategias para el desarrollo de la investigación formativa:

a. Asistentes de investigación, es un espacio para la formación de los estudiantes en la práctica de la investigación, se produce cuando estos colaboran con el profesor que investiga, aprendiendo a formular problemas y proyectos, idear hipótesis, diseñar su metodología, recopilar información, procesar datos, interpretar, inferir y defender resultados, es decir, se aprenden a investigar investigando.

b. Investigación acción educativa, es un método de enseñanza y aprendizaje que fomenta la iniciación de los estudiantes en las actividades propias de la investigación. El docente, emplea la investigación-acción-educativa, centrada en la deconstrucción-reconstrucción de su práctica pedagógica, lo cual le acerca a la investigación formativa; el estudiante, usa como espacio de aprendizaje al proponer problemas, buscar soluciones y comunicar sus resultados.

c. Aprendizaje basado en problemas (ABP), es una estrategia muy eficiente para el desarrollo de la investigación formativa, cuya pertinencia es indiscutible para vincular la educación superior a las necesidades de la sociedad. El ABP favorece la puesta en práctica de la interdisciplinariedad, el diálogo de saberes que es también propio de la investigación moderna. El estudiante se incorpora a la investigación al plantear problemas, revisar la información teórica de base y comunicar los resultados, mediante ensayos, monografías, etc.

d. Enseñanza basada en evidencias, este método se basa en la fase de documentación de todo proceso investigativo. Para diagnosticar y resolver problemas se busca información de casos previos y sus resultados. El estudiante 
interviene en la búsqueda de información teórica o empírica organiza las mismas para elaborar evidencias que contribuyan a resolver los problemas.

e. Aprendizaje cooperativo, es un enfoque interactivo de organización del trabajo en el aula en el cual los estudiantes y sus compañeros son responsables de su aprendizaje; es una estrategia de corresponsabilidad para alcanzar metas e incentivos grupales; fomenta la investigación de temas específicos o tareas grupales.

f. Método de proyectos, este método permite que los estudiantes lleven a cabo la realización de un proyecto en un tiempo determinado para resolver un problema. Este proceso implica la planificación, diseño y realización de una serie de actividades (investigación, exploración, análisis) y todo ello a partir del desarrollo y aplicación de aprendizajes adquiridos con el uso efectivo de recursos didácticos.

g. Estudio de casos, por su naturaleza esta propuesta didáctica, simula bien el proceso investigativo. Parte de un problema por resolver y termina con propuestas plausibles a las que se llega luego de procesos rigurosos de indagación. Al estudiante se le propone un problema en un caso en particular, para lo cual se revisa la información teórica, busca las alternativas de solución y posteriormente, las comunica (Huerta,2017).

\section{Responsabilidad social}

La responsabilidad social es una estrategia de gerencia de los impactos que genera la organización en su entorno humano, social y natural de manera creativa y ética. Lo cual se resume en cuatro impactos definidos por Vallaeys (2016) como ejes de gestión socialmente responsables:

a. Organización y de sus recursos humanos y ambientales.

b. Formación académica y la pedagógica.

c. Producción del saber y los modelos epistemológicos promovidos. d. Participación en el desarrollo humano sostenible de la comunidad.

Pérez (2009), considera que la Responsabilidad
Social Universitaria (RSU), es una nueva manera de funcionamiento de la Universidad, un nuevo modo de hacer y comportarse basado en una relación más fluida y directa de la Universidad con su entorno social, que toma en consideración los efectos, repercusiones y expectativas que la actividad de la Universidad genera tanto en sus propios miembros (docentes, investigadores, personal de administración y estudiantes) como en la Sociedad y su entorno. La RSU exige, desde una visión holística, articular las diversas partes de la institución en un proyecto de promoción social de principios éticos y de desarrollo social equitativo y sostenible, para la producción y transmisión de saberes responsables y la formación de profesionales ciudadanos igualmente responsables (Vallaeys, 2009).

Vallaeys, De la Cruz C. y Sasia (2009), consideran que el proceso de responsabilidad social se desarrolla en cuatro ámbitos de la universidad:

- Ámbito organizacional: la universidad opera en torno a un proyecto universitario, con una estructura que lo desarrolla y unas políticas concretas que lo promueven. Abarca la vida de su personal (administrativo, docente y estudiantil), así como la forma en que organiza su quehacer cotidiano tiene impactos ambientales (desechos, deforestación, transporte, etc.). La universidad responsable se pregunta por su huella social y ambiental. La universidad promueve la gestión organizacional con compromiso social.

- Ámbito educativo: espacio donde la institución se encarga de la formación de sus estudiantes, con una vocación profesionalizante y cívica. La universidad es responsable del tipo de profesionales, ciudadanos y personas que forma, y sobre la adecuada organización de la enseñanza para garantizar $u$ na formación socialmente responsable, por tanto, la universidad promueve la formación social y cívica.

- Ámbito del conocimiento: es responsabilidad de la universidad investigar, producir el saber y transmitirlo. Articula la relación entre tecnociencia y sociedad, posibilitando el control y la apropiación social del conocimiento. Influye en la definición y selección de los problemas del entorno para definir la agenda científica. 
La universidad es responsable del tipo de conocimientos que produce, por su pertinencia social y sus destinatarios. Es, por tanto, la universidad la promotora de la investigación e innovación con responsabilidad social.

\section{- Ámbito social: la universidad como} organización, forma parte de la sociedad e interactúa con otros agentes, colectivos y comunidades, tanto a nivel local como global. Es la responsable de acompañar al desarrollo de la sociedad y ayudar a resolver sus problemas fundamentales. Es en este nivel que la universidad es la organización responsable de fomentar la participación con compromiso social.

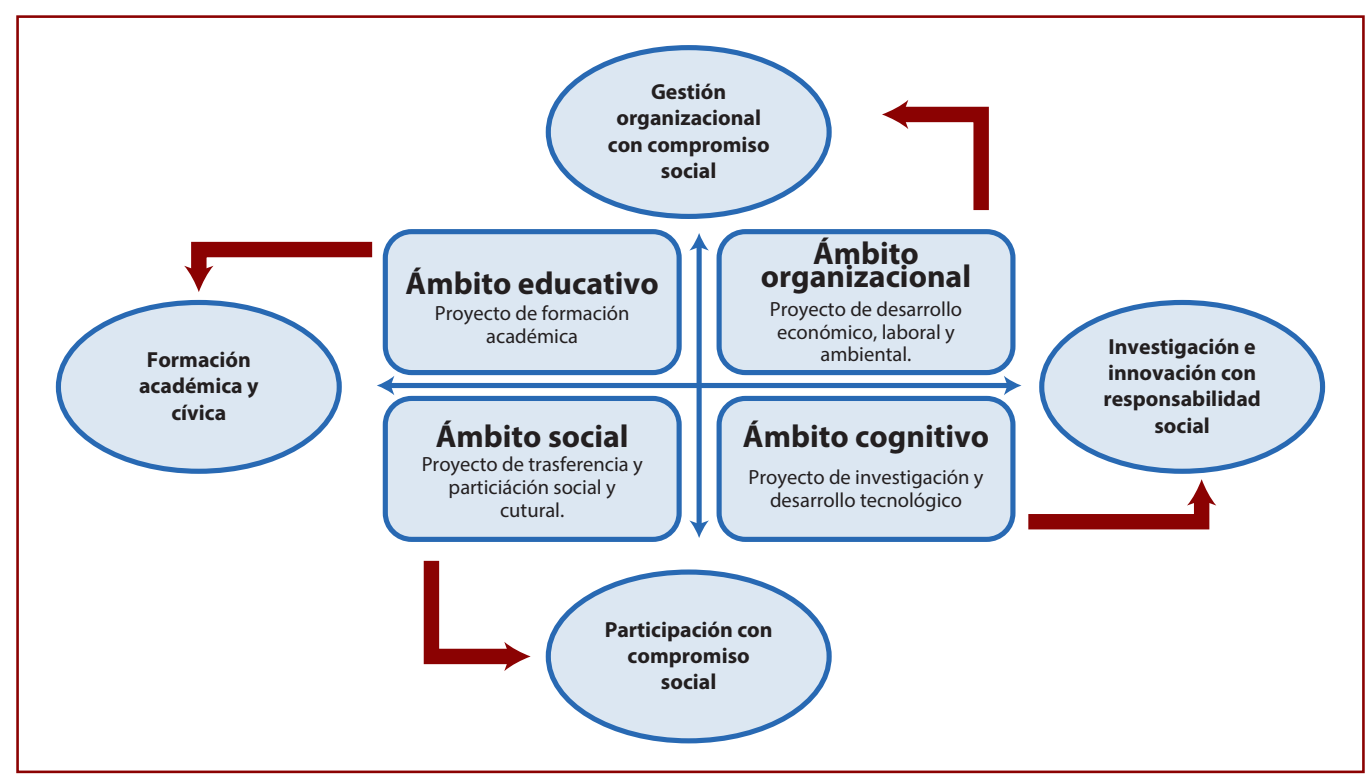

Figura 1. Componentes de la responsabilidad social universitaria.

\section{Estrategias para el desarrollo de la responsabilidad social}

La Responsabilidad Social Universitaria es una política de mejora continua de la Universidad hacia el cumplimiento efectivo de su misión social, señala Vallaeys (2016); para lo cual, se deben trazar las estrategias socialmente responsables para lograr esta mejora, tales como buscar la participación integrada de los grupos de interés internos y externos en el quehacer universitario, articulado de la formación profesional con los planes de estudios, la investigación, la extensión y las estrategias de enseñanza como una forma de resolver problemas de la sociedad. Promoviendo la autoevaluación institucional con herramientas apropiadas de rendición de cuentas hacia los grupos de interés (Vallaeys, 2016)

Por su parte, Pérez (2009) considera que el desarrollo de la RSU se efectiviza a través de cinco fases: a. Diagnóstico: su objetivo es identificar los impactos provocados por la Universidad y confrontarlos con su misión y valores, a fin de diseñar una estrategia para minimizar los impactos negativos y potenciar los positivos. En el diagnóstico deben participar todas las partes interesadas, sean internas o externas a la universidad.

b. Planificación: consiste en la elaboración de un plan de acción detallado que identifique los compromisos, las medidas a adoptar, los cambios en la estructura organizativa que permitan acoger a las partes interesadas, los objetivos a conseguir y las áreas de mejora.

c. Ejecución del plan de acción: puesta en marcha de las acciones con participación de todos los actores.

d. Evaluación de los resultados: es la valoración del impacto, sus aportes y deficiencias a fin de emprender acciones de mejora.

e. Comunicación externa de los resultados: 
esta fase permite generar nuevos argumentos para la realización de un nuevo diagnóstico del funcionamiento y la percepción social de la labor de la Universitaria, que permita iniciar un nuevo proceso, promoviendo la mejora continua de la universidad (Pérez, 2009).

\section{Campos de acción de la responsabilidad social universitaria}

Vallaeys (2016), propone las siguientes acciones: - Apoyar a las facultades y departamentos en la gestión de comunidades de aprendizaje. Es importante precisar que la responsabilidad social universitaria, facilita y da soporte para que los docentes, estudiantes, investigadores y administrativos desarrollen iniciativas y proyectos, en diálogo con actores sociales externos.

- Realizar diagnósticos del estado situacional del entorno de la universidad, para contar con reportes de responsabilidad socioambiental, que involucren a las partes interesadas en el mejoramiento de la sociedad.

- Promover la implementación de estándares, procesos y normas de calidad socialmente responsables dentro y fuera de la Universidad, utilizando las herramientas de Responsabilidad Social Corporativa existentes (Balance Social, Reporte de Sostenibilidad, ISO 14000, AA 1000, SA 8000, etc.), o bien formulando y aplicando herramientas propias.

- Realizar capacitaciones en temas de responsabilidad social para toda la comunidad universitaria (personal administrativo, autoridades, docentes, estudiantes).

- Mantenerse al tanto de las demandas sociales y ambientales a nivel local, nacional e internacional, para plantear nuevas estrategias en la polí tica de responsabilidad social universitaria.

- Comunicar y sensibilizar a la comunidad universitaria interna, así como a socios externos (Empresas, sociedad civil, comunidades, etc.), sobre la necesidad de un $\mathrm{m}$ anejo socialmente responsable de nuestras organizaciones.

- Promover redes universitarias (o académicas en general) de responsabilidad social para incrementar el capital social y el impacto del movimiento de la responsabilidad social universitaria.

\section{Proceso de implementación de la responsabilidad social universitaria}

Vallaeys et al. (2009), proponen un proceso de RSU basado en cuatro pasos.

- Compromiso: Busca articular la RSU con el proyecto institucional, la misión y los valores de la universidad. Exige un claro compromiso de la alta dirección su implicación de toda la comunidad universitaria (autoridades, docentes, estudiantes y administrativos), así como la creación de un equipo que lidere todas las acciones.

- Autodiagnóstico: Permite presentar las herramientas cuantitativas y cualitativas para el diagnóstico de los cuatro ámbitos clave de las universidades (gestión de la organización, formación educativa, la investigación y la participación social).

- Cumplimiento: Tiene como objetivo contrastar los resultados del diagnóstico con la misión de la universidad, planificar las áreas de mejora y ejecutar los proyectos de responsabilidad social, promoviendo la más amplia participación de los miembros de la comunidad universitaria y los actores externos pertinentes.

- Rendición de cuentas: Ofrece algunas ideas para evaluar y comunicar de forma transparente los resultados de los proyectos de mejora institucional, afinar las estrategias y reiniciar el ciclo concentrándose en los aspectos que hayan presentado anteriormente las mayores dificultades.

\section{Proyectos formativos integradores}

Los proyectos formativos para Tobón (2010), son un conjunto de actividades articuladas entre sí, que tienen un inicio, desarrollo y fin, cuyo propósito es abordar un problema personal, familiar, institucional, social, laboral, empresarial, ambiental y/o cultural; para así contribuir a formar una o varias competencias del perfil de egreso. Por tanto, los proyectos formativos son estrategias didácticas y de evaluación de competencias que abordan aspectos comunes a cualquier proyecto, como la contextualización o diagnóstico, planeación, construcción del marco de referencia conceptual, ejecución, evaluación y socialización como parte de la formación profesional. La implementación de proyectos formativos usa la metodología de integración 
curricular, articulando diferentes áreas del conocimiento con los recursos metodológicos para lograr los objetivos desde la complejidad (Morin, 2010).

Tobón (2014), desde la socioformación, considera que los proyectos formativos tienen como característica, resolver problemas del contexto, brindar un servicio o beneficio, ser abordados a través del trabajo colaborativo y permite aprender a gestionar el conocimiento. En conclusión, los proyectos formativos son una estrategia general para formar y evaluar las competencias en los estudiantes mediante la resolución de problemas pertinentes del contexto (personal, familiar, social, laboral-profesional, ambiental-ecológico, cultural, científico, artístico, recreativo, deportivo, etc.) mediante acciones de direccionamiento, planeación, actuación y comunicación de las actividades realizadas y de los productos logrados, a través de la integración de acciones de responsabilidad social e investigación formativa.

\section{Componentes de los proyectos formativos}

Tobón (2014) considera que los proyectos formativos tienen los siguientes componentes:

\section{- Acciones de responsabilidad social}

corresponde a la gestión socialmente responsable de la organización y de sus recursos humanos y ambientales, la formación académica y la pedagógica, la producción del saber y los modelos epistemológicos promovidos y la participación en el desarrollo humano sostenible de la comunidad.

- Desarrollo de la investigación formativa: permite articular diferentes estrategias de investigación disciplinar e interdisplinar en la búsqueda de la información para atender a los problemas del contexto como parte de la formación académica.

- Desarrollo de competencias: en el proceso de formación profesional se conjugan las competencias genéricas y específicas, como propósitos formativos para el desarrollo de las asignaturas a través de las cuales se desarrolla la formación profesional.

- Asignaturas integradas: puede ser dos o más asignaturas que se involucraron en los proyectos formativos integradores y permiten articular las acciones de responsabilidad social con la investigación formativa, para el desarrollo de las competencias genéricas o específicas.

\section{Ventajas de los proyectos formativos en la formación profesional}

La formación profesional se beneficia en los siguientes aspectos con los proyectos formativos integradores:

- Fomenta el aprendizaje pertinente de las competencias: el desarrollo de los proyectos formativos constituye una estrategia didáctica que potencia la formación y evaluación de competencias, potencia el desarrollo en actuaciones integrales para realizar actividades y resolver problemas del contexto con idoneidad y compromiso ético.

- Aprender investigando: el desarrollo de los proyectos formativos permite que los estudiantes aprenden y refuerzan las competencias investigativas, cuando: hacen revisión crítica de la literatura, identificación de problemas; indagación, recolección y análisis de datos; sistematización de la información; etc. En muchos casos los proyectos formativos generan la conformación de semilleros de investigación.

- Genera emprendimientos: a través de los proyectos formativos los estudiantes aprenden a emprender, puesto que mediante estos proyectos se les enseña a planificar e implementar acciones concretas para abordar los problemas en un contexto determinado, y sólo no se queden en la teoría o en el plan de acción. El emprendimiento abarca la dimensión personal, escolar, familiar, comunitario, social, político y empresarial.

- Fomenta el uso de las tecnologías de la información y comunicación: los proyectos formativos permiten al estudiante, utilizar de manera pertinente y comprensiva los diferentes recursos tecnológicos para el desarrollo de todas las actividades y problemas del contexto, y a desarrollar de modo continuo nuevas habilidades de búsqueda, análisis, procesamiento y comunicación de la información.

- Promueve la práctica de valores éticos y morales: la práctica educativa en contextos reales mejora la actuación personal, aprender y reforzar los valores y actitudes, que generan 
retos para la persona en el desarrollo de acciones - actividades diversas en contextos reales que implican el ejercicio de la actuación en contextos sociales, donde se exprese la calidad del desempeño personal. Dichas situaciones deben permitir que los estudiantes aprendan a mejorar su desempeño, autoevaluación y autorregulación, medio fundamental para el desarrollo de la práctica de valores (Tobón, 2012)

\section{Diseño de los proyectos formativos integradores}

Tobón (2010), propone que los proyectos formativos permiten realizar actividades articuladas con los estudiantes para resolver problemas del contexto y así desarrollar las competencias de las asignaturas. Montalván (2016) considera que para implementar la estrategia de los proyectos formativos integradores se debe sustentar en una metodología que debe considerar los siguientes pasos:

a. Definir las asignaturas participantes del proyecto formativo integrador y el nivel de formación en el que se articularán los saberes.

b. Seleccionar las competencias genéricas o específicas y la articulación con las unidades de competencias de las asignaturas que se vincularán al proyecto formativo.

c. Identificar el nodo problematizador como un punto de convergencia o punto de encuentro entre el contexto y las competencias a lograr.

d. Seleccionarel proyecto que se va a implementar, tomando en consideración el impacto que deberá generar en el logro de competencias.

e. Determinar el proceso de implementación del proyecto.

f. Alinear la actuación de los actores del proyecto al proyecto ético de vida.

g. Identificar los estudiantes y docentes a participar, así como las metas a alcanzar y el tiempo a invertir.

\section{Fases de los proyectos formativos integradores}

Las fases de intervención en el proyecto formativo para Tobón (2010) son:

a. Direccionamiento: donde se establecen la meta o metas del proyecto, considerando el aprendizaje o aprendizajes esperados que se tienen en las asignaturas.

b. Planeación: permite establecer qué actividades se van a llevar a cabo en el proyecto.

c. Actuación: explicita las acciones y actividades del proyecto por parte de los estudiantes con el apoyo del docente.

d. Comunicación: los estudiantes informan de los logros, deficiencias y los productos del proyecto (DGEST, 2013).

\section{Finalidad de los proyectos formativos integradores}

Los proyectos formativos integradores tienen como finalidad:

- Fomentar un desarrollo integral, sistémico y holístico del proceso formativo, al integrar los cursos, con sus docentes y estudiantes a actividades de responsabilidad social, involucrándolos en la solución de los problemas del contexto a través de la investigación.

- Se orientan a resolver problemas de carácter social, laboral -profesional, ambiental- ecológico, cultural, científico, cultural, recreativo, deportivo, etc. del contexto.

- Abordan en forma holística la complejidad de la realidad.

- Brindan un beneficio adicional a la formación del futuro profesional, puesto que les permite formarse atendiendo a la solución de los problemas que les aqueja y buscando mejorar su calidad de vida.

- Aportan en la construcción del proyecto ético de vida y de emprendimiento.

En resumen, se puede concluir que los proyectos formativos integradores buscan resolver problemas reales del contexto personal, social, científico, y ambiental, etc., empleando para ello diferentes estrategias y herramienta metodológica para el trabajo curricular; a través de la integración de las asignaturas, agrupadas en ejes temáticos; contribuyen al conocimiento interdisciplinario; articulan teoría-práctica y buscan articular los contenidos disciplinares, científicos y teóricos a la realidad social, ambiental y económica. Para este propósito, se valen de la metodología de gestión de proyectos. 
Así planteados, se pueden implementar:

- Proyectos de salud comunitaria, prevención de enfermedades o cuidado de la salud, orientados a acciones de prevención, organización y atención a problemas de salud como el COVID 19 y otros.

- Proyectos científicos, o tecnológicos orientados al diseño, construcción y transferencia de tecnología (equipos, instrumentos, procesos, entre otros).

- Proyectos sociales, orientados a identificar, diagnosticar y resolver problemas sociales vinculados con la comunidad y su entorno.

- Proyectos ambientales o de salubridad, orientados a la conservación del ambiente y fomentar estrategias de remediación ambiental.

- Proyectos artísticos y culturales orientados a crear o recrear mediante estrategias recreativas, deportivas y artísticas

- Proyectos económicos, orientados a emprender proyectos para generar autoempleos y economías.

\section{Evaluación de los proyectos formativos integradores}

La evaluación de los proyectos formativos integradores, emplea al inicio como evaluación diagnóstica, durante el proceso como evaluación formativa, para valorar el proceso de ejecución implementando estrategias valorativas que deberán ser aplicadas a los docentes yestudiantes, y al final como evaluación sumativa, para evaluar la calidad de los resultados obtenidos y con la finalidad de tomar conciencia de las dificultades, tomar decisiones, reconocer logros y avances, re direccionar los procesos o redefinir objetivos en caso de necesidad; basados en estrategias de evaluación participativas como autoevaluación y coevaluación.

La evaluación de los proyectos formativos integradores se plantea como continua y orientada a detectar problemas de manera oportuna, con la finalidad de mejorar la calidad del proceso formativo, a través del diálogo constante entre docentes y estudiantes para lograr una mejor interacción, que permita a los docentes plantear estrategias de mejoramiento, así como establecer los logros y los desempeños alcanzados por los estudiantes (Montalván, 2016).
¿Qué se evalúa de un proyecto formativo?

- Calidad del diseño del proyecto formativo integrador

- Seguimiento y evaluación de resultados obtenidos

- Evidencias de las etapas del proyecto cumplidas

- Medición de los indicadores ajustados al proyecto

- Sistematización de información sobre el avance y logros de objetivos

- Identificación de competencias y aprendizajes derivados del proceso

- Análisis estadístico de los resultados

- Retroalimentación de actividades y experiencias exitosas y deficientes

- Análisis global de los resultados obtenidos (Tobón, 2010).

\section{APLICACIÓN Y DISCUSIÓN Implementación de los proyectos formativos}

La investigación formativa está alineada con las normas establecidas en la ley universitaria 30220, que en el art. 48, establece que la investigación constituye una función esencial y obligatoria de la universidad, que la fomenta y realiza, respondiendo a través de la producción de conocimiento y desarrollo de tecnologías a las necesidades de la sociedad, con especial énfasis en la realidad nacional. En la misma línea están los estándares de Acreditación del SINEACE, de las condiciones básicas de calidad para el licenciamiento institucional.

Tobón (2010) y otros autores conciben que todo proyecto formativo integrador busca establecer la integración de las actividades de organización y gestión, para intervenir en un contexto problemático y buscar resolverlos. Los cuales se hacen a través de acciones de responsabilidad social articuladas las propias de la investigación formativa, dependiendo de los niveles formativos, por lo que estos deben integrarse desde el plan de estudios de las carreras profesionales y en las herramientas de micro planificación curricular. Para el desarrollo de proyectos formativos se pueden aplicar en tres niveles:

a. Nivel básico. Es la fase de adaptación de los estudiantes y docentes a la intervención social para poder integrar las actividades de 
responsabilidad social que se circunscriben a campañas educativas, campañas de sensibilización, difusión de acciones o medios de intervención entre otros. Por su parte las actividades de investigación formativa inicial implican el desarrollo de actividades de observación, identificación e situaciones problemáticas, el recojo de información, revisión de información científica y la elaboración de informes breves como monografías o informes similares.

b. Nivel intermedio. En esta fase las actividades de responsabilidad social implican una mayor injerencia de los actores en la sociedad, donde se puede observar la intervención en grupos poblacionales, promover su desarrollo o generar la movilización social de grupos poblacionales. Respecto a la investigación formativa se promueve el recojo de información, organización y procesamiento de datos, para elaborar ensayos, informes científicos breves.

c. Nivel superior. En este nivel la responsabilidad social implica la posibilidad de que estudiantes y docentes generen el desarrollo de proyectos productivos o la generación de actividades de trasferencia tecnológica comprometiendo a la sociedad. Por su parte, la investigación formativa, permite generar la formulación y desarrollo de proyectos de investigación, elaborar trabajos de campo, redactar informes científicos o publicarlos.

Para este propósito se ha diseñado la siguiente matriz que permita la vinculación entre la formación profesional, investigación formativa y responsabilidad social, el cual debe tener la siguiente estructura:

- Programa, que señale alguna propuesta de probables áreas de intervención con la finalidad de resolver un problema, fomentar el desarrollo social, económico, ambiental, cultural, etc., en un contexto sociocultural determinado. El programa puede incluir varios proyectos.

- Proyecto, describe la viabilidad de una actividad prevista ser desarrollada en un tiempo involucrando a docentes y estudiantes en acciones de responsabilidad social e investigación formativa.

- Actividades de RSU, describe las acciones de responsabilidad social universitaria previstas a ser desarrolladas como parte del proyecto.

- Actividades de investigación formativa, describe las acciones o tareas propias de investigación formativa que implican el desarrollo de técnicas procedimientos de recojo de información, organización y comunicación de los resultados.

- Asignaturas integradas, se debe detallar las asignaturas involucradas en cada uno de los proyectos a desarrollar. 
Tabla 1

Proyectos formativos integradores en el plan de estudios

\begin{tabular}{|c|c|c|c|c|}
\hline Programa & Proyecto & $\begin{array}{l}\text { Actividades de } \\
\text { RSU }\end{array}$ & $\begin{array}{l}\text { Actividades de } \\
\text { investigación } \\
\text { formativa }\end{array}$ & $\begin{array}{l}\text { Asignaturas } \\
\text { integradas }\end{array}$ \\
\hline \multirow{2}{*}{$\begin{array}{l}\text { Educación } \\
\text { ambiental }\end{array}$} & $\begin{array}{l}\text { Campañas de } \\
\text { educación } \\
\text { ambiental }\end{array}$ & $\begin{array}{l}\text { Señalización con } \\
\text { carteles } \\
\text { educativos }\end{array}$ & $\begin{array}{l}\text { - Diagnósticos } \\
\text { situacionales } \\
\text { - Elaboración de } \\
\text { monografías }\end{array}$ & $\begin{array}{l}\text { - Comunicación oral } \\
\text { y escrita } \\
\text { - Realidad nacional } \\
\text { y mundial }\end{array}$ \\
\hline & $\begin{array}{l}\text { Campañas de } \\
\text { limpieza rivereña }\end{array}$ & $\begin{array}{l}\text { Campaña de } \\
\text { sensibilización } \\
\text { para recojo de } \\
\text { desechos }\end{array}$ & $\begin{array}{l}\text { - Revisión de la } \\
\text { literatura } \\
\text { - Elaboración de } \\
\text { ensayos }\end{array}$ & $\begin{array}{l}\text { - Liderazgo } \\
\text { - Educación } \\
\text { ambiental }\end{array}$ \\
\hline \multirow[t]{2}{*}{$\begin{array}{l}\text { Alimentación } \\
\text { saludable }\end{array}$} & $\begin{array}{l}\text { Campaña de } \\
\text { recuperación de } \\
\text { nuestros alimentos } \\
\text { tradicionales }\end{array}$ & $\begin{array}{l}\text { Campaña de } \\
\text { difusión de } \\
\text { alimentos andinos }\end{array}$ & $\begin{array}{l}\text { - Diagnósticos } \\
\text { situacionales } \\
\text { - Elaboración de } \\
\text { monografías }\end{array}$ & $\begin{array}{l}\text { - Biología } \\
\text { - Ecología } \\
\text { - Desarrollo } \\
\text { personal y social }\end{array}$ \\
\hline & $\begin{array}{l}\text { Campaña de } \\
\text { difusión de dietas } \\
\text { saludables }\end{array}$ & $\begin{array}{l}\text { Campaña "Come } \\
\text { sano" }\end{array}$ & $\begin{array}{l}\text { - Revisión de la } \\
\text { literatura } \\
\text { - Publicación de } \\
\text { informes } \\
\text { científicos }\end{array}$ & $\begin{array}{l}\text { - Realidad nacional } \\
\text { y mundial } \\
\text { - Ecología } \\
\text { - Biología }\end{array}$ \\
\hline \multirow[t]{2}{*}{$\begin{array}{l}\text { Salud } \\
\text { comunitaria }\end{array}$} & $\begin{array}{l}\text { Campaña de } \\
\text { prevención al } \\
\text { COVID } 19\end{array}$ & $\begin{array}{l}\text { Campaña } \\
\text { "Quédate en casa" }\end{array}$ & $\begin{array}{l}\text { - Elaboración de } \\
\text { monografías } \\
\text { - Reporte de } \\
\text { casos }\end{array}$ & $\begin{array}{l}\text { - Biología } \\
\text { - Realidad nacional } \\
\text { y mundial } \\
\text { - Desarrollo } \\
\text { personal y social }\end{array}$ \\
\hline & & $\begin{array}{l}\text { Campaña usa bien } \\
\text { la mascarilla y la } \\
\text { distancia social }\end{array}$ & $\begin{array}{l}\text { - Elaboración de } \\
\text { monografías } \\
\text { - Reporte de } \\
\text { casos }\end{array}$ & $\begin{array}{l}\text { - Biología } \\
\text { - Desarrollo } \\
\text { personal y social }\end{array}$ \\
\hline
\end{tabular}

El desarrollo de los proyectos formativos integradores, permite que se promueva la capacidad de resolver problemas del contexto, para ello se puede brindar un servicio o proceso o producto en beneficio de la comunidad o la universidad; el cual debe ser abordados a través del trabajo colaborativo y permitir aprender a gestionar el conocimiento por equipos de docentes, administrativos y estudiantes; para que, entre ellos, formulen un proyecto común que permita la vinculación entre la formación profesional, la investigación formativa y la responsabilidad social. El propósito final, es articular la formación profesional con su desempeño un contexto real; a través de acciones concretas de responsabilidad social, investigación formativa como estrategias de aprendizaje.

La implementación de la investigación formativa, es entendida como la identificación de problemas, revisión de la literatura, formulación de hipótesis, recojo y procesamiento de la información y la comunicación de los mismos en poblaciones relevantes para estructurar o refinar sus proyectos de investigación.

Con los trabajos académicos se busca 
proporcionar a los estudiantes un conjunto de técnicas y procedimientos para la búsqueda, recopilación y selección de la información, así como el análisis y procesamiento de datos y la comunicación de sus resultados, que implique el desarrollo de procesos de investigación.

Son de naturaleza formativa e instrumental y debe permitir al estudiante, ir adquiriendo habilidades investigativas. Los cuales concluyen generalmente con los diferentes trabajos académicos como son: la monografía, el ensayo, el informe técnico, la tesina o el artículo científico. Los proyectos de investigación formativa se desarrollarán en cada una de las carreras profesionales donde se recomienda establecer un sistema de investigación formativa que fomente la capacidad de observación, recojo de información, análisis y procesamiento de datos, búsqueda y selección de información científica primaria, establecer los esquemas básicos para la comunicación de los trabajos académicos, según el avance académico.

Por ejemplo:

$\begin{array}{ll}\text { Trabajo académico } & \text { Ciclos académicos } \\ \text { Monografía } & \text { A partir del I } \\ \text { Ensayo } & \text { A partir del III } \\ \text { Informe técnico } & \text { A partir del IV } \\ \text { Artículo científico } & \text { A partir del VI }\end{array}$

La implementación de la responsabilidad social responde al desempeño de la comunidad universitaria (estudiantes, docentes y personal administrativo) a través de la gestión responsable de los impactos educativos, cognitivos, laborales y ambientales que la Universidad genera, en un diálogo participativo con la sociedad para promover el Desarrollo Humano Sostenible (Vallaeys, 2010).

A través de la responsabilidad social universitaria se promueve la formación de estudiantes agentes del desarrollo humano sostenible de su país, que sepa gestionar adecuadamente los impactos de la Universidad (laboral, ambiental, educativo, cognitivo y social), producir conocimientos socialmente útiles, abrir la universidad hacia la sociedad y la sociedad hacia la universidad, y crear comunidades de aprendizaje para el desarrollo, con participación de docentes, estudiantes, administrativos y actores sociales externos a la Universidad.

En el silabo se pueden desarrollar actividades como:

- Un programa de educación ambiental en una institución educativa.

- Charlas de prevención de desastres a una comunidad.

- Talleres de construcción de cocinas mejoradas - Campañas de salud preventiva.

- Asesoramiento técnico en el manejo financiero a los pequeños comerciantes y otros.

Tabla 2

Proyectos formativos integradores en el silabo

\begin{tabular}{|c|c|c|c|}
\hline Proyecto & Actividades de RSU & $\begin{array}{l}\text { Actividades de investigación } \\
\text { formativa }\end{array}$ & $\begin{array}{l}\text { Asignaturas } \\
\text { integradas }\end{array}$ \\
\hline $\begin{array}{l}\text { Campañas de } \\
\text { educación } \\
\text { ambiental }\end{array}$ & $\begin{array}{l}\text { Señalización con } \\
\text { carteles educativos }\end{array}$ & $\begin{array}{ll}\text { - } & \text { Revisión de la literatura } \\
\text { - } & \text { Diagnósticos } \\
& \text { situacionales } \\
\text { - } & \text { Elaboración de } \\
\text { - } & \text { monografías } \\
\text { - } & \text { Publicación de informes. }\end{array}$ & $\begin{array}{ll}\text { - } & \text { Comunicación } \\
\text { oraly escrita } \\
\text { - } & \text { Realidad } \\
& \text { nacional y } \\
& \text { mundial } \\
\text { - } & \text { Desarrollo } \\
& \text { personal y social }\end{array}$ \\
\hline $\begin{array}{l}\text { Campañas de } \\
\text { limpieza rivereña }\end{array}$ & $\begin{array}{l}\text { Campaña de } \\
\text { sensibilización de } \\
\text { recojo de desechos }\end{array}$ & $\begin{array}{ll}\text { - } & \text { Revisión de la literatura } \\
\text { - } & \text { Diagnósticos } \\
& \text { situacionales } \\
\text { - } & \text { Elaboración de ensayos } \\
\text { - } & \text { Publicación de informes. }\end{array}$ & $\begin{array}{ll}\text { - } & \text { Liderazgo } \\
\text { - } & \text { Educación } \\
& \text { ambiental } \\
\text { - } & \text { Desarrollo } \\
& \text { personal y social }\end{array}$ \\
\hline
\end{tabular}




\section{CONCLUSIONES}

1. La aplicación de los proyectos formativos integradores en la formación profesional de universitaria, potencia la capacidad de investigar, puesto que prepara al estudiantes en el empleo de herramientas de diagnóstico, búsqueda de información, procesamiento y análisis de tatos, como la comunicación de los resultados a la comunidad académica; por su parte integrado a ello aprende a contribuir a la solución de los problemas del contextos a través de los proyectos de responsabilidad social, permitiendo con ello a la formación del ser humano investigador con responsabilidad humana, social y ambiental.

2. En todo proyecto formativo se integra la investigación formativa como herramienta estratégica en la formación de estudiantes investigadores, la responsabilidad social, fomentando la toma de conciencia de los problemas del contexto y cómo intervenir en la solución de los mismos; permitiendo integrase en proyectos de desarrollo integral que contribuyen a formar estudiantes con compromiso social y agentes de cambio de la sociedad.

\section{REFERENCIAS BIBLIOGRÁFICAS}

Ander Egg, E. (2001). Técnicas de investigación social. Buenos Aires: Hvmanitas. Disponible en: $\quad$ https://epiprimero.files.wordpress. com/2012/01/ander-egg-tecnicas-deinvestigacion-social.pdf

Cassany, D.; Luna, M. y Sanz, G. (2008). Enseñar lengua. Grao: Madrid. Disponible en: http://lenguaydidactica.weebly.com/ uploads/9/6/4/6/9646574/cassany,_d._ luna,_m._sanz,_g._-_ensenar_lengua.pdf

Concytec (2017). Política Nacional para el Desarrollo de la Ciencia, Tecnología e Innovación Tecnológica - CTI. Lima: Concytec. Disponible en: http://portal. concytec.gob.pe/index.php/concytec/ estrategias/628-politica-nacional-para-eldesarrollo-de-la-ciencia-la-tecnologia-y-lainnovacion-tecnologica-cti
DÍAZ, A. (1999). Aproximación al texto escrito. Medellín: Universidad de Antioquia.

DGEST (2013). Proyectos integradores para el desarrollo de competencias profesionales del SNIT. México. Secretaria de Educación Pública. Disponible en: http://www. ittoluca.edu.mx/difusion/Proyectos\%20 Integradores\%20Toluca.pdf

Guerra, M (2010). Manual de publicaciones de la American Psychologiical Association, tercera edición. México: Editorial manual Moderno.

Hernández R., Fernández C. y Baptista P. (2016). Metodología de la investigación. Sexta edición. México: Mc Graw Hill.

Huerta M. (2017). Formación por competencias a través del aprendizaje estratégico. Lima: Editorial San Marcos.

Kerlinger F.N. y Lee H. (2002). Investigación del comportamiento. Métodos de investigación. México: McGraw Hill.

Montalván, M. (2016). Aprendizaje basado en proyectos formativos integradores como metodología de integración curricular. Tesis maestría, Universidad Católica deSantiago de Guayaquil. Disponible en: http://repositorio. ucsg.edu.ec/bitstream/3317/7236/1/TUCSG-POS-MES-61.pdf

Moreno, F.; Marthe N.; Rebolledo L. (2010). Cómo escribir textos académicos según normas internacionales: APA, IEEE, MLA, Vancouver e ICONTEC. Barranquilla: Ediciones Uninorte. Disponible en: https://josedominguezblog. files.wordpress.com/2015/06/comoescribir-textos-academicos-segun-reglasinternacionales.pdf

Morillo, D. (2016). El proceso innovador. Disponible en: http://gerenciaconstruccion.blogspot.com/2016/02/ elproceso-innovador-daniel-e.html 
Morin, E. (2010). Los siete saberes para la educacióndelfuturo.MinisteriodeEducación. Disponible en : https://ideassonline. org/public/pdfLosSieteSaberesNecesariosParaLaEdudelFuturo.pdf

Padilla J., Rincón, D. y Buitrago L. (2015). La investigación formativa desde la teoría de las representaciones sociales en la Facultad de Estudios a Distancia de la Universidad Militar Nueva Granada. Revista Academia y Virtualidad 8(1): 21-34, 2015. Disponible en :https://dialnet.unirioja.es/descarga/ articulo/5104744.pdf

Pérez F. (2009). La responsabilidad social universitaria (RSU). Consejo Social de la Universidad de Huelva. Disponible en : http://rabida.uhu.es/dspace/bitstream/ handle/10272/13428/RSU\%20con\%20 bibliograf\%20 - \% 20U HU\% 202009 . pdf?sequence $=2$

Restrepo, B. (2008). Formación Investigativa e investigación Formativa: Acepciones y Operacionalización de esta última y Contraste con la Investigación Científica en Sentido Estricto. Disponible en : http:// web.unap.edu.pe/web/sites/default/files/ Formacion-investigativa-e-Investigacionformativa.pdf

Salkind N. (2010). Métodos de investigación Científica. México: McGraw Hill.

Sánchez, A (2011). Manual de redacción académica e investigativa: cómo escribir, evaluar y publicar artículos. Medellín: Católica del Norte Fundación Universitaria. Disponible en : https://www.ucn.edu. co/institucion/sala-prensa/documents/ manual-de-redaccion-mayo-05-2011.pdf

Tobón S. (2010). Secuencias didácticas, aprendizaje y evaluación de competencias. Pearson Educación. Disponible en : http:// files.ctezona141.webnode.mx/2000000048ed038fca3/secuencias-didacticastobon120521222400-phpapp02.pdf
Tobón, S. (2012). Proyectos formativos y desarrollo de Competencias. Disponible en: $\quad$ https://www.researchgate.net/ publication/314246215

Tobón, S. (2012). Proyectos formativos. Ejes claves. Centro Universitario CIFE. Disponible en: En https://issuu.com/ cifeformacionporcompetencias/docs/ ebook_proyectos_2

Vallaeys, F. (2009). ¿Qué es la Responsabilidad Social Universitaria?. Disponible en: http://creasfile.uahurtado.cl/RSU.pdf

Vallaeys, F., De la Cruz C. y Sasia, P. (2009). Responsabilidad social universitaria: manual de primeros pasos. Mcgraw-Hill Interamericana

Vallaeys, F. (2016). Introducción a la Responsabilidad Social Universitaria (RSU) oEdiciones Universidad Simón Bolívar. Disponible en: http://publicaciones. unisimonbolivar.edu.co/edicionesUSB/ dptopublicaciones@unisimonbolivar.edu. co

Varón, C. (2017). Gestores bibliográficos [Recurso electrónico]: recomendaciones para su aprovechamiento en la academia. Medellín: Journals \& Authors.Disponible en: https://jasolutions.com.co/wp-ontent/ uploads/2017/04/GetoresBibliograficos.pdf 